Methods We compared DR resuscitation practices (obtained from the neonatal database) of infants born between $25+0$ and $28+6$ weeks gestation at RWH between 2003-2006 and 20072012.

Results 1013 infants were included in the study, 97\% of which received respiratory support in the DR during the ten-year period (Figure 1). Mean gestation (SD) and birth weight (SD) were $27+0$ (1.11) weeks and 947(244) grams, respectively. There was a substantial decrease in the number of newborns intubated in the DR between 2003-2006 (58\%) and 2007-2012 $(47 \%)(p=0.05)$. The number of infants intubated in the DR remains highest at lower gestational ages.

Conclusion There has continued to be an increase in the use of CPAP instead of intubation in the DR since completion of the COIN trial, suggesting a change in clinical practice.

\section{P0-0696 BASELINE COAGULATION TIMES DO NOT INFLUENCE LIKELIHOOD OF INTRAVENTRICULAR HAEMORRHAGE (IVH) IN EXTREMELY PREMATURE NEONATES}

${ }^{1}$ E Neary, ${ }^{2} \mathrm{~F}$ Ni Ainle, ${ }^{2} \mathrm{M}$ Cotter, ${ }^{1} \mathrm{~N}$ McCallion. ${ }^{1}$ Paediatrics, Rotunda Hospital, Dublin, Ireland; ${ }^{2}$ Haematology, Rotunda Hospital, Dublin, Ireland

10.1136/archdischild-2014-307384.1335

Background Derangements of haemostasis are implicated in IVH. Prothrombin time (PT), activated partial thromboplastin time (APTT) and fibrinogen level are frequently monitored in premature infants. Neonates frequently receive frozen plasma (FP) in attempt to correct perceived haemostatic abnormalities based on laboratory results.

Methods Prospective observational study was performed. Blood was drawn into citrated tubes from neonates $(<30 / 40)$ on admission $(n=76)$ from non-heparinised lines. Platelet poor plasma was obtained by centrifugation of whole blood; PT, APTT, and fibrinogen were measured and correlated with IVH.

Results Infants with IVH ( $\mathrm{n}=30)$ had no significant difference in PT $(\mathrm{p}=0.949)$, APTT $(\mathrm{p}=0.405)$ and fibrinogen $(\mathrm{p}=$ $0.560)$ than those without IVH $(n=46)$. There was no association between IVH grade and APTT ( $p=0.937)$. There was no significant difference in APTT in those with or without IVH, excluding infants with IVH on admission ( $p=0.534)$. Of patients administered FP, there was no significant difference in IVH $(p=0.38)$. FP is frequently administered when APTT $>100$ s. In this subgroup; IVH rates in those that received FP (n $=17)$ vs. those that did not $(n=4)$ was not significantly different $(p=0.447)$. There was no significant difference in IVH development in high risk (APTT $>100 \mathrm{~s}$, Administered FP) vs. low risk infants (APTT $<100 \mathrm{~s}$, No treatment), $(\mathrm{p}=1.00)$ or when comparing infants with lesser degrees of coagulopathy (APTT $60 \mathrm{~s}-80 \mathrm{~s}$ vs. $80 \mathrm{~s}-100 \mathrm{~s}, \mathrm{p}=0.6334$ ).

Conclusions Justification of FP based on coagulation values is unclear. In this study, IVH rates following FP administration was not increased. Coagulation values were not predicative of IVH, indicating lack of therapeutic window for intervention.

\section{PO-0697 AN EDUCATIONAL INTERVENTION TO IMPROVE NURSE'S UNDERSTANDING OF PAIN IN CHILDREN}

${ }^{1}$ AR Dongara, ${ }^{1}$ SM Nimbalkar, ${ }^{2}$ AG Phatak, ${ }^{1}$ DV Patel, ${ }^{3}$ AS Nimbalkar. 'Department of Pediatrics, Pramukhswami Medical College, Karamsad, India; ${ }^{2}$ Central Research Services, Charutar Arogya Mandal, Karamsad, India; ${ }^{3}$ Department of Physiology, Pramukhswami Medical College, Karamsad, India

10.1136/archdischild-2014-307384.1336
Background and aims Accurate assessment of pain and its management is important but challenging aspect of paediatric care. Nurses, usually the primary care-givers showed inadequate knowledge and restrictive attitudes towards pain assessment. We evaluated feasibility of an educational intervention to improve the situation.

Methods Workshops targeted at overall understanding of pain, its assessment and management strategies were conducted for nurses working in paediatric/neonatal wards and intensive care units and paediatric cardiac intensive care unit. A modified and consensually validated "Knowledge and Attitudes Survey Regarding Pain questionnaire-2008" consisting 25 True/False questions, 8 Multiple Choice Questions and 2 case scenarios was administered before, immediately after and 3 months after the workshops to evaluate the impact of the intervention. Descriptive statistics, paired t test and test of proportion were used to depict the results.

Results Eighty-seven (all females) nurses participated in the study. Mean (SD) age and experience was 27.7(6.4) and 4.04 (5.9) years respectively. About half (49.4\%) of the nurses had not previously heard of pain scales while $47.1 \%$ reported using a pain scale in their routine practice and 37\% felt they could assess pain without any scale. A statistically significant improvement was observed between the pre-test and post-test total score (15.69[2.94] vs. 17.51[3.47], $\mathrm{p}<0.001)$ as well as the pre-test and retention score (15.69[2.94] vs. 19.40[4.6], $\mathrm{p}<0.001)$.

Conclusions The educational intervention was successful and better retention test scores suggest cascading effect. Pain assessment and management in children should be incorporated in the nursing curriculum and should be reinforced in all paediatric units.

\section{PO-0698 NEONATAL RESUSCITATION PRACTICES IN THE DELIVERY ROOM IN INDIA: AN AUDIT USING VIDEOGRAPHY}

SM Nimbalkar, DV Patel, AS Nimbalkar, AR Dongara, AG Phatak, R Vasa. Department of Pediatrics, Pramukhswami Medical College, Karamsad, India

\subsection{6/archdischild-2014-307384.1337}

Background and aims Care given to a newborn in the first few minutes of life following delivery is protocol driven. Yet there are deviations which do occur. Deviations may be due to unrealistic expectations from the protocol. We audited practices of managing patients in relation to guidelines for neonatal care.

Methods Observational Study. Continuous video recording occurred at the warmer where babies are routinely placed after delivery. The videos were downloaded and analysed as per steps related to neonatal resuscitation guidelines 2010.

Results In all the 77 videos assessed, pre-delivery arrangements were made appropriately and in time. The post- delivery care was provided by a single person in $24(31.2 \%)$ cases. In most cases this was provided by first year residents (53) and nurses (34). Wearing gloves, providing routine care, drying the baby and changing the wet sheet was followed in $100 \%$ cases. The mean (SD) time required to provide routine care was 6.97 (2.07) minutes. Heart Rate was not assessed in $30(39 \%)$ cases whereas Respiratory Rate was not assessed in 28 (36.4\%) cases. The mean (SD) time at which heart are and respiratory rate was assessed were 6.31 (9.17) and 4.16 (1.68) minutes. PPV was required in just one case and it was done properly.

Conclusions Fairly short duration of routine care was provided to neonates with absence of major issues. However regular 